\title{
Subrelativistic Jets' Heating Mechanisms
}

\author{
Yuri Krivosheyev* \\ Space Research Institute (IKI) 84/32 Profsoyuznaya str., 117997 Moscow, Russia \\ E-mail: krivosheeveiki.rssi.ru
}

\section{Gennady Bisnovatyi-Kogan}

Space Research Institute (IKI) 84/32 Profsoyuznaya str., 117997 Moscow, Russia

National Nuclear Research University "MEPHI" 31 Kashirskoye ave., 115409 Moscow, Russia

E-mail: gkogan@iki.rssi.ru

\begin{abstract}
Different physical processes, influencing the X-ray jet's thermal balance are considered. We focus on the problem of possible fast jet cooling via radiative losses. Thus, the contributions of each process to the jet thermal balance are calculated. We investigate possible jet heating by inverse Compton scattering of hard X-ray quanta on jet electrons, the influence of shock wave propagation on jet heating, and the mechanism of jet kinetic energy transformation into heat via Coulomb collisions of jet and corona protons. Quantitative estimates are made for the case of Galactic microquasar SS433 based on previous results of the authors. The only important heating mechanism for this source turned out to be Coulomb collisions.
\end{abstract}

25th Texas Symposium on Relativistic Astrophysics - TEXAS 2010

December 06-10, 2010

Heidelberg, Germany

*The work was supported by RFBR grant 08-02-00491, by the Russian President's grant "Leading scientific school" NSh-8784.2010.1. The work of YuK was partially supported by the Russian President's grant for support of young scientists MK-8696.2010.2 and by the Russian Science Support Foundation. 


\section{Introduction}

Jets are quite frequent in the Universe and can have various sizes and shapes. Despite the fact that they were discovered decades ago, many questions regarding them remain unanswered. The main issues deal with jet formation, the mechanisms of jet collimation and heating. In the present paper we will focus on the last issue that requires jet thermal balance equation investigation. We consider the X-ray jet, i.e. the region close to the base of the jet, fully ionized, with temperatures around tens $\mathrm{keV}$. Under these conditions the major energy loss mechanisms are bremsstrahlung and adiabatic expansion cooling. Jet temperature profile strongly depends on the bremsstrahlung to adiabatic losses ratio. In the case of SS433 we consider quite dense jet, surrounded by substantially less dense corona, emitting hard X-rays. It was demonstrated in [1] that the best fit of simulations to INTEGRAL data gives us jet to corona density ratio about 200 with only adiabatic cooling considered. But bremsstrahlung cooling at the bottom of the jet is several times greater than adiabatic cooling (about 3.5 times greater). In the absence of jet heating mechanisms it must lead to rapid jet cooling shifting the X-ray jet boundary closer to the bottom, in contradiction with observations. So, mechanisms supplying energy to the jet must be present to compensate for radiative cooling. Here we investigate three possible jet heating mechanisms: 1. Inverse Compton scattering of hard corona X-rays on jet electrons, 2. Heating by shock wave energy dissipation generated at the bottom of the jet, 3. Heating by jet kinetic energy transformation into heat via jet and corona protons Coulomb collisions. Our calculations show the importance of the third mechanism in supporting the jet thermal balance only.

\section{Jet energy balance equation}

Let us write jet internal energy balance equation assuming that it consists of mono atomic perfect gas. This approximation is well justified in the X-ray jet, where the temperature is high enough to maintain full ionization, gas is rarefied enough to neglect particle interaction and radiation energy is small because of small optical depth in the transversal direction.

$$
\frac{d \varepsilon}{d t}=q-p \frac{d V}{d t}
$$

Here $\varepsilon$ denotes internal energy per unit mass $q$ - energy inflow/outflow per unit mass, $p$ - gas pressure, $V=1 / \rho$ - volume per unit mass. Because we assume that gas flow is stationary, and in every point gas moves in radial direction with velocity $v_{\text {jet }}=0.27 c$, we have $d / d t=v_{\text {jet }} d / d r$. Internal energy of a perfect gas with $\gamma=5 / 3$ is related to density and pressure via $\varepsilon=3 p / 2 \rho$, pressure is related to temperature via $p=n k T$ ( $n$-particle concentration, $k$ - Boltzmann's constant). We neglect electron contribution to density $\rho=m_{p} n$ and use density profile of a stationary conic flow with constant opening angle and mass flow $n_{j e t}=n_{j e t, 0}\left(r_{0} / r\right)^{2}, n_{\text {jet }, 0}$ is defined by jet opening angle and mass flow ratio. For SS433 it was shown in [1] that the best fit to observations correspond to $n_{\text {jet }, 0}=0.86 \cdot 10^{15} \mathrm{~cm}^{-3}$. We will use this value in our calculations. For bremsstrahlung cooling we will accept a known expression $q^{f f}=0,852 \cdot 10^{-3} n_{\text {jet }} \sqrt{T}$ (see, e.g. [2]). The equation (2.1) will transform into an equation on jet temperature

$$
\frac{d T}{d r}=-\frac{4 T}{3 r}-\frac{2 m_{p}}{3 k v_{j e t}} q^{f f}
$$




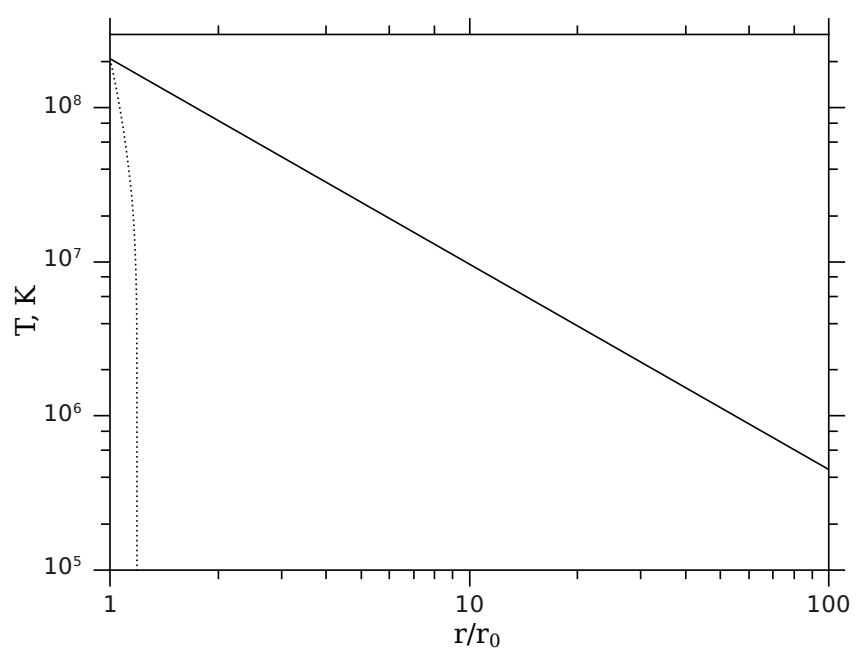

Figure 1: Jet temperature profiles with adiabatic cooling only (solid line) and bremsstrahlung cooling included (dotted line).

The temperature at the base of the jet $T\left(r_{0}\right)=T_{0}$ is set to be equal $18 \mathrm{keV}$ (see [1]). Introducing new variables $t=T / T_{0}$ and $x=r / r_{0}$ ( $r_{0}$ is the distance to jet base) and expressing bremsstrahlung cooling in terms of radiative losses $q^{f f}\left(r_{0}\right)$ to adiabatic cooling $q^{a d}\left(r_{0}\right)=-2 v_{j e t} k T / m_{p} r_{0}$ ratio $D$ at jet base we obtain the following equation for dimensionless temperature profile:

$$
\frac{d t}{d x}=-\frac{2}{3}\left(2 \frac{t}{x}+D \frac{\sqrt{t}}{x^{2}}\right), t(1)=1,
$$

The equation (2.3) was investigated in [3], they obtained an analytical solution in the form

$$
\frac{T}{T_{0}}=\left(\frac{r_{0}}{r}\right)^{4 / 3}\left\{1-D\left[1-\left(\frac{r_{0}}{r}\right)^{1 / 3}\right]\right\}^{2}
$$

The case with $D \ll 1$ corresponds to small radiative losses compared to adiabatic ones and the temperature profile does not differ significantly from the case or pure expansion. We have a different case here - $D=2 q^{f f} / q^{a d}\left(r_{0}\right) \approx 7,6$, so the jet will cool dramatically and the X-ray jet boundary will be much closer to the jet base than it appears in observations. Fig. 1 illustrates the problem.

The radiative cooling temperature profile was obtained with bremsstrahlung (free-free transitions) cooling only. In our calculations we will use a more realistic radiative cooling curve based on analytical approximation [4] of numerical calculations of [5-7] with bound-free and bound-bound transitions included. The obtained radiative cooling temperature profile contradicts observational data, according to which the X-ray jet extends up to $100 r_{0}$ [8]. It is evident that some mechanisms supplying energy to the jet must be present to compensate for radiative cooling.

\section{Contribution of Comptonization to the jet's thermal balance}

The jet matter interacts with photon gas consisting of accretion disk emission and corona bremsstrahlung. We are interested in the X-ray jet region with temperatures around $1 \mathrm{keV}$, so the matter and emission will interact mainly via Compton scattering. The new frequency of a 
photon with frequency $v$ being scattered on a free electron moving with velocity $\beta c$ (Lorentz factor $\gamma=1 / \sqrt{1-\beta^{2}}$ ) will be [9]

$$
v^{\prime}=v \frac{1-\mu \beta}{1-\mu^{\prime} \beta+h v / \gamma m_{e} c^{2}(1-\cos \phi)},
$$

where $\mu$ and $\mu^{\prime}$ are cosines of angles between directions of motion of photon and electron in the lab frame before and after scattering respectively, $\phi$ being the angle between initial and final photon directions of motion (scattering angle) in the lab frame. We neglect photon frequency change due to recoil effect compared to Doppler shift change. At the jet base the electron thermal velocity is comparable to the bulk velocity $\left(\beta_{t h} \sim 0.3 c\right)$, so the electron distribution is not very different from the the isotropic case and Comptonization cools the electrons. As jet matter cools down the thermal component of jet velocity becomes small and Comptonization begins to heat the jet as the photons create chaotic electron momentum component via scattering, i.e. increase their temperature. To estimate the frequency change in both cases we set the relative frequency change equal to

$$
\frac{\Delta v}{v} \approx \beta
$$

We estimate the value of Compton heating/cooling. The amount of energy gained/lost by photons from/to unit mass of jet matter via Compton scattering is given by the expression:

$$
q_{C} \approx \frac{\sigma_{T} c}{m_{p}} \int d v h \Delta v \frac{\varepsilon_{v}}{h v}=3 \sigma_{T} \beta n_{0, c o r} r_{c o r} q_{0, c o r}^{f f}\left(\frac{r_{0}}{r_{c o r}}\right)^{3}\left[1+\frac{\varepsilon^{d i s k}}{\varepsilon^{c o r}}\right]
$$

$\sigma_{T}=6.65 \cdot 10^{-25} \mathrm{~cm}^{2}$ - Thomson scattering cross-section $n_{0, \text { cor }}$ - corona density at its base, $r_{\text {cor }}$ - corona radius, $\varepsilon^{d i s k}$ and $\varepsilon^{c o r}$ - disk and corona photon densities respectively. Using the expression for bremsstrahlung cooling we obtain the Comptonization cooling/heating to radiative cooling ratio:

$$
\frac{q_{C}}{q_{0, j e t}^{f f}}=3 \sigma_{T} r_{\text {cor }} \frac{n_{0, \text { cor }}^{2}}{n_{0, j e t}} \sqrt{\frac{T_{0, c o r}}{T_{0, j e t}}} \beta\left(\frac{r_{0}}{r_{\text {cor }}}\right)^{3}\left(1+\frac{\varepsilon^{\text {disk }}}{\mathcal{\varepsilon}^{\text {cor }}}\right) \ll 1,
$$

If we take into account that $n_{c o r, 0} / n_{\text {jet }, 0}=0.005, r_{0} / r_{\text {cor }}=0.15$, and the values $\varepsilon^{\text {disk }} / \mathcal{E}^{\text {cor }}$ and $3 \sigma_{T} r_{c o r} n_{0, c o r} \beta \sqrt{\frac{T_{0, c o r}}{T_{0, j e t}}}$ are both the order of unity, we conclude that Compton cooling is negligible with respect to radiative cooling. So, we will not take this effect into account when investigating jet thermal balance.

\section{Heating by shock wave energy dissipation}

Shock waves can be generated at the jet bottom by turbulent or convective motions. We will assume that a shock wave propagates along the jet generated at its bottom $r=r_{0}$ with given Mach number $M_{0}$. In the jet rest frame the shock wave front propagates with velocity $D=M c_{s}$, where $c_{s}$ is the local sound speed in the unperturbed gas. We have a stationary state, so we will assume a sequence of shock waves separated by given time interval. We will characterize this state by 'average' temperature $T$ for the time interval $\Delta t$. The value of $\Delta t$ can be inferred from observations. 


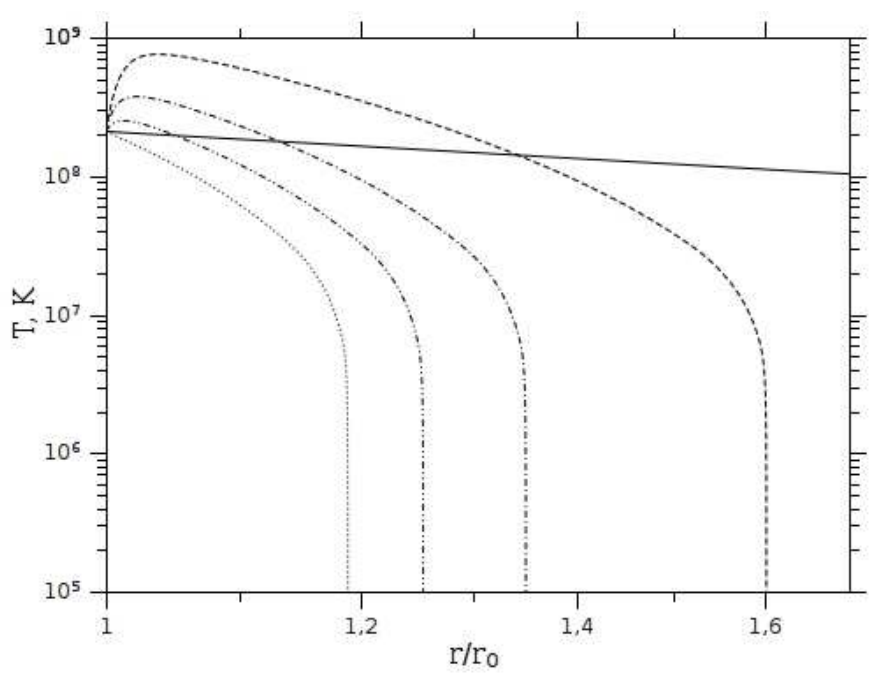

Figure 2: Jet temperature profiles with shock wave heating included. Dashed line corresponds to shock wave propagation with $M=2$, dash-dotted - to $M=3$, dash-dot-dotted - to $M=5$. Dotted line - temperature profile without heating, solid line - adiabatic cooling only.

For SS433 we set it to be equal to 1 second based on X-ray time variability observations [8]. The mean shock wave heating can be approximated as

$$
\begin{gathered}
q_{s w} \approx \frac{T \Delta S}{\Delta t}= \\
\frac{k T}{(\gamma-1) m_{p} \Delta t}\left[\ln \left(\frac{2 \gamma M^{2}}{\gamma+1}-\frac{\gamma-1}{\gamma+1}\right)+\gamma \ln \left(\frac{\gamma-1}{\gamma+1}+\frac{2}{M^{2}(\gamma+1)}\right)\right]
\end{gathered}
$$

Shock energy dissipates into heat, so we write shock wave energy density balance equation governing the local Mach number evolution:

$$
\begin{gathered}
\frac{2\left(M^{2}-1\right)}{(\gamma+1)^{2}} \rho_{0} c_{s}^{3}\left[\left(3+\frac{1}{M^{2}}\right) \frac{d M}{d r}+\frac{1}{M}\left(M^{2}-1\right)\left(\frac{1}{\rho_{0}} \frac{d \rho_{0}}{d r}+\frac{3}{2 T} \frac{d T}{d r}\right)\right] \\
=-\rho_{0} \frac{(\gamma+1) M^{2}}{2+(\gamma-1) M^{2}} q_{s w}
\end{gathered}
$$

$\rho_{0}$ is the unperturbed by shocks density profile in the jet. We introduce the shock wave heating term in the jet energy balance equation and obtain the equation for temperature profile:

$$
\frac{1}{\gamma-1} \frac{d T}{d r}=\frac{T}{\rho_{0}} \frac{d \rho_{0}}{d r}+\frac{m_{p}}{k}\left(\frac{q_{s w}}{M c_{s}}-\frac{q_{b r}}{v_{j e t}}\right) .
$$

The solution of the last two coupled equations will yield temperature and Mach number profiles in the jet (Fig.2)

It is clear that shock waves can heat the jet locally, near the place of generation. To heat the whole jet we need a system of shocks generated along the jet because of broad spectrum of initial perturbations or because of jet matter interaction with the surrounding medium. The last mechanism was mentioned in [10] to explain the heating of SS433 X-ray jet. 


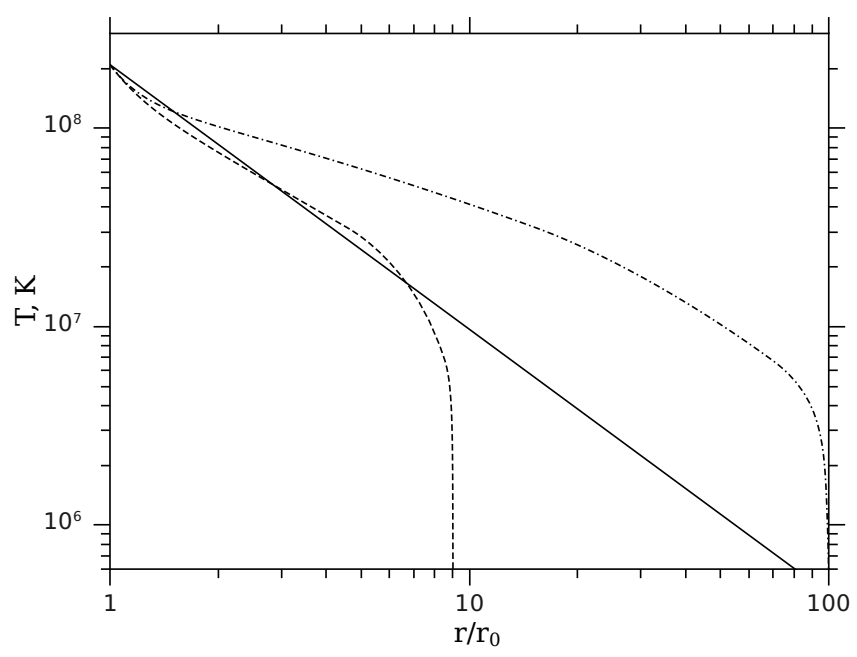

Figure 3: Jet temperature profile with radiative cooling and collisional heating included. $a=\left(r / r_{0}\right)^{-3 / 2}$ (dashed line), $a=\left(r / r_{0}\right)^{-1.163}$ (dash-dotted line). Solid line - adiabatic expansion case.

\section{Proton Coulomb collisions}

Let us consider jet heating by Coulomb collisions of corona and jet protons. Thermal corona protons enter the jet where they serve as targets for jet protons moving at $v_{j e t}=0.27 c$. As a result of scattering, jet protons lose part of their energy to corona protons thus transforming jet kinetic energy into heat. We estimate the maximum heating rate that can be produced by this mechanism as the number of protons with Maxwell distribution crossing unit surface per unit time $n v_{t h} / 4$ $\left(v_{t h}=\sqrt{8 k T / \pi m}\right)$ multiplied by jet proton kinetic energy $m_{p} v_{j e t}^{2} / 2$ :

$$
q_{\text {coll }, \max }=v_{\text {jet }}^{2} \frac{v_{\text {th }}}{2 d_{\text {jet }}} \frac{n_{\text {cor }}}{n_{\text {jet }}} \sim \frac{1}{r}
$$

Here conical jet shape is assumed $d_{\text {jet }}=2 \operatorname{rtg}\left(\theta_{\text {jet }} / 2\right)$ and corona and jet density profiles in the form $n_{\text {jet }}=n_{\text {jet }, 0}\left(r_{0} / r\right)^{2}, n_{\text {cor }}=n_{\text {cor }, 0}\left(r_{0} / r\right)^{2}$ with $n_{\text {cor }, 0}=4.3 \cdot 10^{12} \mathrm{~cm}^{-3}, n_{\text {jet }, 0}=0.86 \cdot 10^{15} \mathrm{~cm}^{-3}$ [1]. Then collisional heating to radiative cooling ratio at a distance equal to corona radius $r \approx 6,4 r_{0}$ is maximal and equal to $\approx 34$. This is the upper limit for $q_{c o l l} / q_{b r}$ because it is assumed that all jet kinetic energy is transformed into heat. Actually, jet is thin for corona protons with respect to Coulomb scattering, so collisional heating will reduce significantly if corona proton trajectory is straight line. But if magnetic field is present, proton trajectories are curved and they stay longer in the jet, increasing the effectiveness of collisional heating. Magnetic field of $\approx 1.3 \cdot 10^{-4} \mathrm{G}$ is enough for proton gyroradius to be equal to jet radius. In reality, magnetic field value $B_{j e t 0}$ is several orders of magnitude greater $B_{\text {jet }} \gg B_{\text {jet } 0}$, jet is thick for corona protons and collisional heating is effective. We do not know how magnetic field does vary with distance along the jet, so to model magnetic field radial dependence we introduce function $a(r)$ characterizing the fraction of maximal hating rate in the collisional heating term.

$$
q_{\text {coll }}(r)=q_{\text {coll }, \max }(r) * a(r)
$$


The results of calculations with collisional heating are presented in Fig.3. It is clear from the figure that Coulomb collisions can heat the X-ray jet effectively. When jet temperature drops to $\approx 10^{7} \mathrm{~K}$ rapid cooling occurs because of significant increase of radiative losses. The dashed line in the figure follows the adiabatic curve quite well up to distances of about $10 r_{0}$ explaining the X-ray observations [1], the dash-dotted line corresponds to excessive heating of the X-ray jet. Both cases require $<10^{37} \mathrm{erg} / \mathrm{s}$ kinetic energy conversion which is less than $1 \%$ of the jet kinetic luminosity.

\section{Conclusion}

It was demonstrated that with radiative cooling taken into account in the jet energy balance equation using data obtained in [1] leads to fast jet cooling with distance. We have investigated three possible jet heating mechanisms. Comptonization does not contribute much to the energy balance. Shock wave, propagating along the jet heat it locally, their energy is dissipated in a small interval close to their generation place. The whole jet can be heated by a system of shocks generated along the jet by broad spectrum of initial perturbations or by interaction of jet matter with the surrounding medium. The most promising mechanism of jet heating is jet kinetic energy transformation into heat by Coulomb collisions of jet and corona protons. In the presence of small magnetic field of about $\sim 0.1-1 \mathrm{G}$ effective heating of the while $\mathrm{X}$-ray jet region can be achieved requiring only a small fraction of jet kinetic energy flux (less than 1\%). Because of that jet bulk motion speed decrease with distance due to heating is too small to be observable at the moment. More details about the calculations can be found in [11].

\section{References}

[1] Yu. M. Krivosheyev, G. S. Bisnovatyi-Kogan, A. M. Cherepashchuk, K. A. Postnov, Monte-Carlo simulations of the broadband X-ray continuum of SS433 Mon.Not.R.Astron.Soc. 394 (2009) 1674.

[2] G. S. Bisnovatyi-Kogan Physical Problems in the Theory of Stellar Evolution, Nauka, Moscow 1989 (in Russian) [English translation: Stellar Physics, Vol. 1, 2. Springer, Berlin 2001]

[3] E. V. Koval', N. I. Shakura, in proceedings of 23rd ESLAB Symp. on Two-Topics in X-Ray Astronomy, Bologna, Italy, 13-20 September, 1989, ESA SP-296 (1989).

[4] M. V. Barkov G. S. Bisnovatyi-Kogan, Astron.Zhurn. 82 (2005) 8, 1 (in Russian).

[5] D. P. Cox, W. H. Tucker, Astrophys.J. 157 (1969) 1157.

[6] J. C. Raymond, D. P. Cox, B. W. Smith, Radiative cooling of a low-density plasma Astrophys.J. 204 (1976) 290.

[7] P. R. Shapiro, H. Kang, Astrophys.J. 318 (1987) 32.

[8] S. Fabrika, Astrophys.Sp.Phys.Rev., 12 (2004) 1.

[9] Space Physics (small encyclopedia) edt. R. A. Sunyaev (2nd edition) Soviet Encyclopedia, Moscow 1986 (in Russian)

[10] H. Marshall, C. Canizares, N. Schulz, in proceedings of Conference Chandra's First Decade of Discovery, Boston, USA, 22-25 September, 2009, abstract No.149.

[11] G. S. Bisnovatyi-Kogan, Yu. M. Krivosheyev Jet heating mechanisms with application to microquasar SS433 Astron.Zhurn. (2011) (submitted) 